\title{
On the Classification of Diseases
}

\section{Introduction}

Identifying the necessary and sufficient conditions for individuating and classifying diseases is a matter of great importance in the fields of law, ethics, epidemiology, and of course medicine. In this paper I first provide a means of individuating diseases, ensuring that no two distinct disease-types could correctly be ascribed to the same disease-token. I then posit a metaphysical ${ }^{1}$ ontology of diseases - that is, I give an account of what a disease is. This is essential to providing the most effective means of interfering with disease processes.

Following existing work in the philosophy of medicine and epidemiology (primarily Boorse; Whitbeck; Broadbent), philosophy of biology (LaPorte 2004; Hull 1987), conditional analyses of causation (JL Mackie 1974; Lewis 1973), and recent literature on dispositional essentialism (Mumford and Anjum 2011; Bird 2007), I endorse a dispositional metaphysics of disease (DMD) whereby (i) diseases are individuated by their causes, and (ii) diseases are causal processes best seen as simultaneously acting sequences of mutually manifesting dispositions - this, I claim, follows from the assumption that diseases should be classified by consideration of both their clinical and pathological effects, and importantly, events that can lead to the cessation of these effects.

\section{The Ontological Conception}

Whenever possible, the first objective in any medical examination is to determine what disease (if any) a patient is suffering from, as this inevitably contributes to prognosis and treatment. One attempt to individuate diseases has been labelled the 'ontological conception' (OC), whereby in order to distinguish one disease from another one (merely) has to distinguish between the kinds of pathogen (Whitbeck refers to pathogens as 'disease-entities') lodged in the patient (Whitbeck, 1977, 623), as the identity of the disease is fixed entirely by the entity causing the patient's symptoms ${ }^{2}$ - but this is problematic for a number of reasons. Consider the WHO system of influenza virus nomenclature (which comprises two parts):

\footnotetext{
${ }^{1}$ To illustrate the distinction between conceptual and metaphysical analysis as I intend it, one can look to Hume's work on causation. Hume began his conceptual enquiry by considering the collision of billiard balls, concluding that there are three aspects to the concept of causation: contiguity in space and time, constant conjunction, and necessary connection. Bearing his conceptual analysis in mind, Hume went on to provide one of the most influential metaphysical analyses in modern philosophy. He explained the nature of 'necessary connection' in causation, and what we were really referring to when we used the term in our conceptual and everyday causal talk. I do not pretend to do anything so revolutionary here (!), but the reasoning process in this paper is similarly structured - the conceptual precedes the metaphysical analysis of disease and its related concepts.

${ }^{2}$ Note that according to this view the disease is not the invading pathogen itself (a virus in a petri dish is certainly not a disease). The ontological conception tells us that when a patient is suffering from a disease, the
} 
(a) a type and strain designation, and (b) for influenza A viruses, a description of the antigenic specificity (subtype) of the surface antigens ( $\mathrm{H}$ and $\mathrm{N}$ ). The strain designation for influenza virus types $A, B$ and $C$ contains information on the antigenic type of the virus (based on the antigenic specificity of the nucleoprotein), the host of origin (for strains isolated from non-human sources), geographical origin, strain number, and year of isolation...' (WHO, 1980)

According to $\mathrm{OC}$, two strains of a virus cause the same disease only if they are of the same kind (since the virus fixes the identity of the disease), but in order to identify one case of a disease with another, we must know the right level of kind-specificity the viruses must both be members of (The Titanic and The Beagle are members of the same kind at the level of boats, but not at the lower level 'kinds of boat'). Take the etiological agents for flu symptoms to be the strains of virus that cause (and under the ontological account, in a sense are) the disease. The influenza viruse(es) can differ in many non-trivial respects: H1N1 A/England/i/51 (HiNi) is a different strain of influenza virus from H3N2 A/Hong Kong/1/68 (H3N2). These two strains of influenza, however, often bring about similar symptoms on their host. Patients suffering similar flu symptoms might receive similar treatments, and though the subtypes of the virus differ, they closely resemble one another genetically at some low-order levels of specificity. We might think two agents, one suffering from the influenza A H1N1 virus and one from the influenza A H3N2 virus are suffering from the same disease. To classify them as distinct diseases by their subtypes thus seems too fine-grained, so using the higher order 'influenza A' (or perhaps even 'influenza') kind is prima facie more appropriate.

Figure 1.

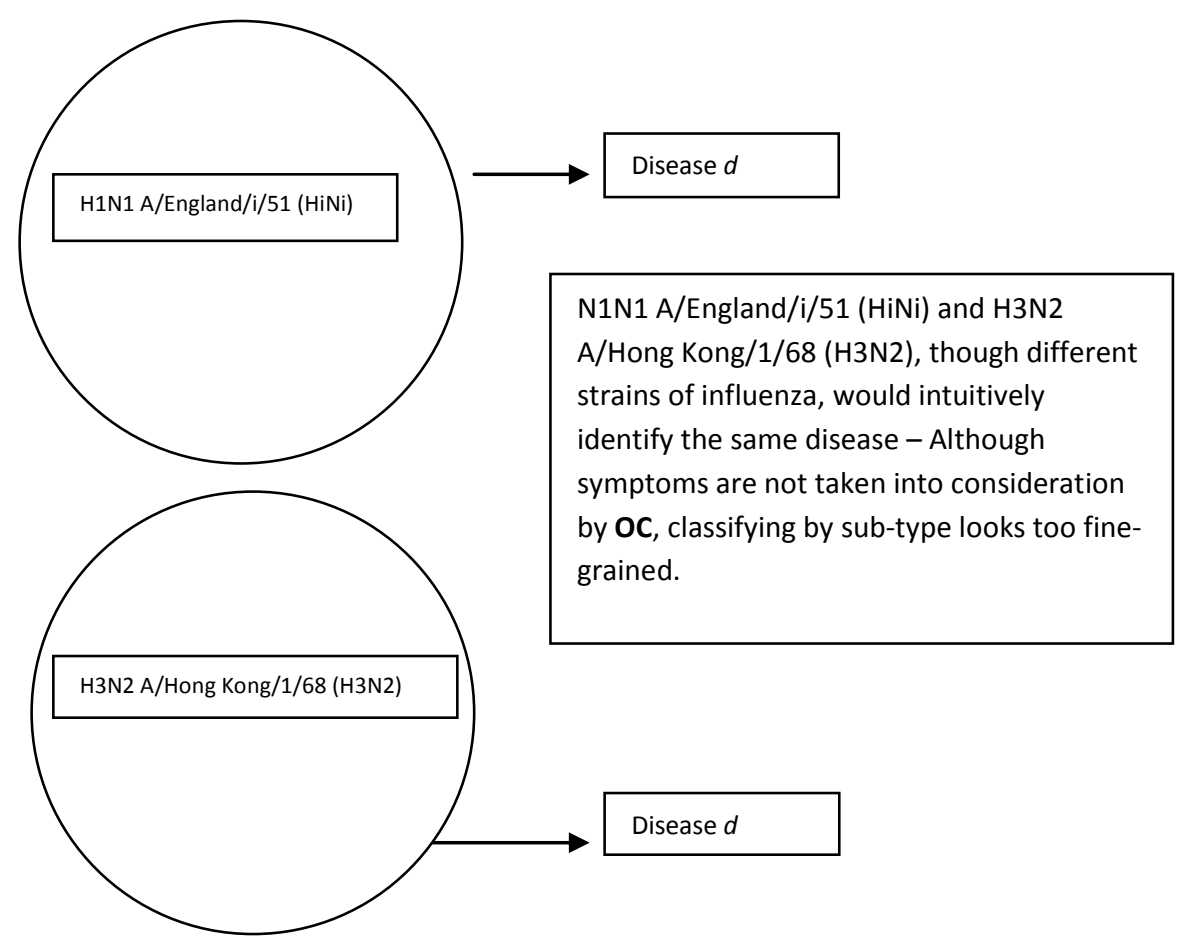

disease he is suffering from can be individuated purely by identifying the pathogen lodged in the patient. The pathogen causing disease $d$ fixes the identity of $d$. The pathogen itself is not the disease. 
However, one type (type 6) of human papillomavirus (HPV) can be a contributing factor to laryngeal papillomatosis, anal lesions, anogenital warts, and oral papillomas (amongst other ailments). Different types of HPV virus are also associated with a variety of cancers (Muñoza et al, 2006, ch 1), and recent studies have even suggested the virus can cause cardiovascular disease (Kuo and Fujise, 2001, 6). It would be strange to suppose that these were all the same disease purely on the grounds that they originate from the same general type, HPV. Therefore using classifications of virus as broad as 'influenza' (or even 'influenza A') is unsuccessful. Indeed, even at a very fine-grained level the symptoms suffered as a result of viral infection often differ substantially. Despite the strong similarities, strains of H5N1 avian flu, for example, can differ in ways other than the type of hemagglutinin and neuraminidase on the virus's surface. These differences can give rise to different amino acid sequences at the hemagglutinin cleavage sites, which in turn determine whether or not the strain is low-pathogenic avian influenza (LPAI) or highly pathogenic avian influenza (HPAI). Low pathogenic strains are of little concern ${ }^{3}$, but recent outbursts of highly pathogenic $\mathrm{H} 5$ and $\mathrm{H} 7$ avian flu viruses have caused global panic (due more to the possibility of a pandemic (post-mutation) in the human population, than to a shortage of chickens!). Given the extreme difference in mortality rates it surely cannot be right to suppose that two birds, one with an LPAI and one with an HPAI strain of H5N1 strain of avian flu, are suffering from the same disease. On the face of it, then, the confliction concerning the level at which we should classify a disease as 'of the same type' arises (at least partly) from the similarities and differences in symptoms. To resolve the issue it seems these differences must also be taken into consideration, but OC does not allow symptomatic differences to be a contributing factor, as it is ex hypothesi grounded purely in terms of the entity lodged in the patient causing these symptoms.

One might think the issue could be resolved by classifying diseases at the fundamental level - that is, two diseases are one and the same if and only if the viruses causing their symptoms are (near) physical duplicates. However, because the symptoms suffered by a patient are a function not only of the virus but also of the patient, and environmental factors, often different patients will suffer in such different ways that we could not classify them as having the same disease. As one and the same virus can manifest itself in many different ways (such that the diseases suffered are quite clearly distinct, even if related by one of their causes) depending on the patient and their circumstances, one cannot identify a case of a disease purely by knowing its 'invading' etiological agent. OC fails.

\section{Classification by Causes}

Here I propose a model of 'causal classification of disease' (CCD). This is not a metaphysical account of what a disease is, but an account of how to individuate one disease from another (once we know that we are distinguishing diseases, rather than some other entity). The cause of a disease is not the disease itself, of

\footnotetext{
${ }^{3}$ Insofar as LPAI strains do not have high mortality rates. There are reasons to keep track of LPAI strains as they can evolve into HPAls.
} 
course - CCD merely provides a means of individuating diseases. In section 4 I provide a metaphysics of disease that further motivates classifying diseases in terms of their causes.

CCD looks open to many of the same objections as OC (as often the cause is considered to be an invading micro-organism), but it is far less problematic ${ }^{4}$. Consider a case where the HPV virus causes patient to manifest genital warts as a young woman, and at a later date the virus was deemed to be the cause of her cervical cancer. Although the two distinct diseases are caused by the same etiological agent, one can see that the invading organism does not tell the whole causal story. When a patient contracts flu $Z$ one might determine the contraction of the influenza virus $X$ to be the cause. However the virus is not the only factor involved in the patient contracting the disease. She has had a particularly poor immune system since she became HIV positive $Y$. This contributed to her contracting the flu, as ceteris paribus without $Y$ her immune system would have been strong enough for her to fight off the infection. Neither $X$ nor $Y$ is individually sufficient for $Z$, but both are causally relevant and jointly sufficient. Despite their being individually insufficient, we might nonetheless consider the 'interesting' etiological agent to be $X$, or $Y$, or both, depending on what information we consider most salient.

Figure 2

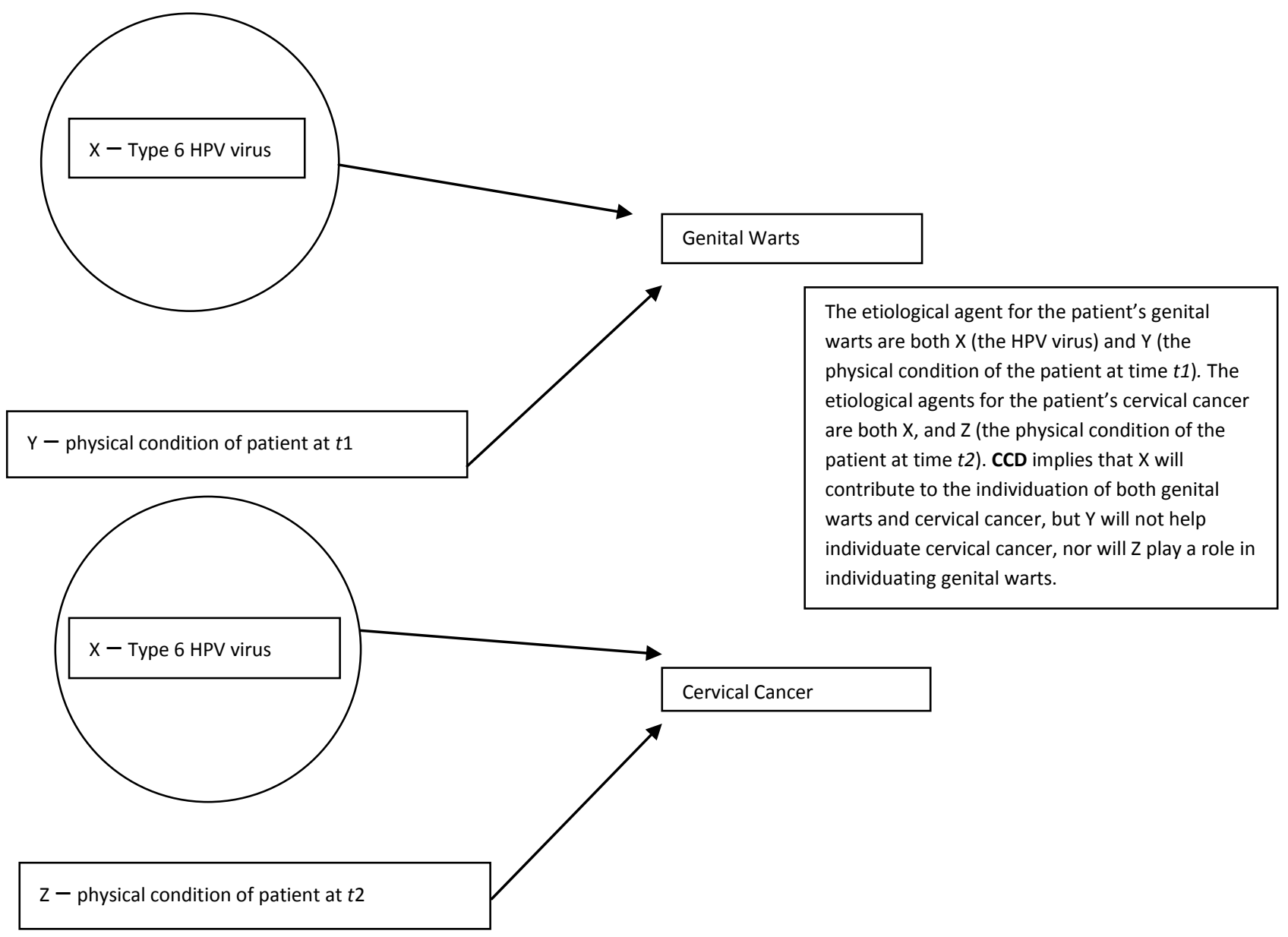

\footnotetext{
${ }^{4}$ Note that the ontological account is intended to be both a means of individuating diseases and an account of what a disease is
} 
One might disagree, and suggest we should designate $X$ the cause on the grounds that (unlike $Y$ ) $X$ is a necessary condition for Z. Alex Broadbent labels the thesis that "every disease has a necessary and circumstantially sufficient cause, and by its logic ensures (or near enough for practical purposes) that it will have at most one such cause" the monocausal model (MM) (Broadbent, 2009, 302), whilst pointing out that there are real causal differences between different instances of the same disease. Consider HPV and cardiovascular disease (CVD) (and suppose there's a genuine causal connection between HPV and CVD). Assuming we endorse Kuo and Fijise's (2011-11-01) findings, HPV is the cause (or one of the causes) of token cases of CVD, but being infected with HPV is by no means a necessary condition for getting CVD. Carrying the HPV virus is thus neither necessary, nor sufficient for CVD. Furthermore, as we saw with the flu patient, there being more than one cause of the same instance of a disease should not be ruled out.

According to the multifactorial accounts, there are generally numerous causal conditions ${ }^{5}$ associated with a disease which are only jointly sufficient for it. Furthermore, there are likely to be numerous distinct jointly sufficient conjunctions of causal conditions. When we consider what the causes of cardiovascular disease are (on a more general scale), then, we have a disjunction of conjunctions of causal conditions - CVD results from 'XY or QY or....' Where '...' represents a finite number of conjunctive conditions; we may assert that CVD is always preceded by 'XY or QY or...', and conversely that all 'XY or QY or...' are followed by CVD. Furthermore, an effect might also be partially reliant on the absence of certain occurrences. In the CVD case, for example, lots of exercise might ' $\mathrm{mask}^{6}$ the disease. These are welcome additions to the causal classification model, as many diseases, such as scurvy and rickets, are the result of a lack of something (in these cases, vitamin $\mathrm{C}$ and vitamin D deficiencies respectively). The result picture looks like this: 'Z iff XYnot-D or QYnot-D or...' ${ }^{7}$. Note that some philosophers (notably Schaffer 2005; Broadbent 2012) take selection to be part of the concept of causation, whereby only those events we would normally pick out as causes in the context can be contenders. However the method of classification I presuppose requires a 'broad and non-discriminatory concept of causation' (Lewis, 1973, 557) such as that ably defended by David Lewis (Lewis 1973; 2000).

Given the removal of 'selection' from the process of cause-identification, the exhaustive list of conditions to be satisfied for most effects (for our purposes these are diseases, but this is a general account of causation) is enormous. Mill writes, though, that contrary to our every day conception 'the cause...philosophically speaking, is the sum total of the conditions positive and negative' ((Mackie, J.L. 1974, 63) quoting J.S. Mill). Following Mackie, let us call Mill's 'philosophically speaking' cause the 'full cause'. According to CCD a disease is

\footnotetext{
${ }^{5}$ For now, consider a causal condition to be an event causally relevant to the contraction of the disease in question.

${ }^{6}$ 'Mask' is used here in Johnson's (1992) sense - exercise might mask a patient's disposition to get CVD just as dampening a match masks its disposition to light when struck.

${ }^{7}$ This resultant picture forms an integral part of J.L. Mackie's (1974) conception of singular causation, where Mackie identifies a cause as any 'insufficient but non-redundant part of an unnecessary but sufficient condition' (an inus condition), that is, any one condition to be found within what he terms, the 'full cause'.
} 
identified by its full cause, that is, the disjunction of conjunctions of events jointly sufficient for contracting the disease.

Pragmatically speaking, the 'full cause' is clearly not what we refer to in standard causal talk, either within or outside of an epidemiological/medical context. When we identify causes in a medical context the salient information is that which leads to diagnosis, prognosis, explanation, and the identification of suitable treatments for the disease. Indeed, a medic need not even identify precisely what disease a patient is suffering from in order to (non-accidently) prescribe the correct treatment. Many antibiotics, for example, will successfully treat a large number of different bacterial infections, so in many cases to help a patient the medic need only identify the infection as bacterial. The salient cause(s) (where 'cause' is being used in the 'every-day' sense of the term - that is, one of the conditions in one of the conjuncts that partly comprise our full-cause) and corresponding treatment(s) are thus often associated with many different diseases, which (at least in part) accounts for the failure of $\mathbf{M M}$ - even if there is only one salient cause, it is often insufficient to individuate the contracted disease. CCD looks more promising than this, as once we take the cause to be the cause qua 'cause philosophically speaking', no two diseases will have the same full cause.

In this section I have described a means of individuating one process, or one process-type, from others. This says nothing of which processes count as diseases and which do not. In this paper I do not endeavour to resolve this matter, but in section $5 \mathrm{I}$ demonstrate that the metaphysics of disease I endorse is compatible with both kinds of contender.

\section{Objections $i$}

Mackie's general account of causation implicitly assumes that our conceptual framework is deterministic, but it's not obvious that a conceptual account of disease individuation can be so. Epidemiology is rife with talk of chance: "chance of infection", "chance of survival", "chance of going into remission", and so on.

Response: 'Chance', in medical contexts, tends to refer to statistical probability, whereby one's assigned 'chance of survival/recovery etc' is determined by the percentage survival rate of similar patients with similar ailments, at similar stages of the disease, in similar environmental conditions, and so on. Statistical probability talk does not rule out deterministic conceptions of causation. Indeed 'frequentist' versions of deterministic probability, although generally rejected on a naïve 'probabilities-equate-to-actual-frequencies' level, have been popular with many philosophers (Reichenbach 1949; Salmon 1967, Lewis 1994; Cohen and Callender 2009), so although the debates here are beyond the scope of this paper, 'smoking raises the (statistical) probability of getting lung cancer' and 'lung cancer has its identity fixed by a (deterministic) full cause' look to be compatible claims. There are also possible ways of amending the account to allow for real chance (that is, allowing that even upon knowing all states of affairs at $t$ and all the laws of nature, it would be impossible to know for certain the state of affairs at $t+1$ due to objective probabilities in nature). One can give probabilityraising accounts of causation, where the causal conditions are such that they are necessary to raise the 
probability of the effect in the circumstances. This would, in fact, be sufficient for my thesis. However, here I am happy to endorse metaphysical determinism and frequentist accounts of probability.

\section{Objection ii}

Different stages of a disease have different 'full causes', and so CCD would identify them as different diseases.

Response: The model identifies diseases by the causal conditions of their initial contraction. Any stage of a disease could be traced back to the conjunction of events that caused the first stage of the disease - CCD individuates a disease by the full cause only of its first stage (that's not to say that a later stage of a disease cannot be individuated by the full cause of a disease-stage, but this is a virtue of the model).

\section{Objection iii}

Assuming determinism and the transitivity of causation, the cause of any token disease $D$ in person $P$ is also the cause of any subsequent illness suffered by $P$. Suppose $P$ has infectious disease $D$ which weakens her immune system, and as a result she contracts a more serious illness $D^{*}$ - how does one distinguish between $D$ and $D^{*}$. Assume that for all practical purposes it is best to treat $D$ and $D^{*}$ as distinct diseases. According to CCD, how does one distinguish between $D$ and $D^{*}$ as distinct diseases, when $D$ and $D^{*}$ might be seen as different stages of the same disease with the same cause, as discussed in objection ii?

Response: The aim here is to find a means of individuating diseases, that is, for no two diseases to satisfy the same conditions - CCD succeeds in this regard. CCD does not tell us which full causes identify diseases and which do not, but if $D$ and $D^{*}$ are distinct diseases (which we have agreed they are), then they each have their own unique full cause. Although it raises an important issue, objection iii does not defeat CCD.

\section{Objection iv}

"...if all we say about diseases is that they have many and diverse causes, it is not clear what more we can hope for than a catalogue. And the catalogue will never be complete, because in practice the causes of even the most ordinary event are beyond human counting" (Broadbent, 2009, 308). Here Broadbent makes the point that the full cause (or something like it) cannot be used in general explanation, and as diseases are "kinds of ill health for which general explanations are available (ibid) a catalogue of causes is an inadequate means of classifying disease.

Response: I agree with the motivations for Broadbent's objection. We classify diseases for practical purposes, primarily in the hope that we can better cure and/or prevent ill-health. To do this we first need the model to provide general explanations for ill-health, but does CCD fail in this regard? Broadbent claims that a model of disease purely comprised of lists of causes does not explain why we differentiate between different 
diseases, 'it says nothing about what disease is, nor about why we distinguish between diseases in the way that we do ${ }^{8}$. However in token cases (and here I speak of causal sequences in general, not just those concerning disease), causal explanations of particular events can be very useful. With token cases of ill-health, causal explanations can undeniably help us determine which procedures should be followed to restore good health ${ }^{9}$. So why not generalise this claim, that is, from the cause of a disease-token to the full cause of a disease-type? If we identify a disease by its causes, we explain the ill-health - diagnosis becomes the identification of causal explanation (more specifically, the identification of the range of possible causal explanations), and points towards possible treatment(s) for the disease-type diagnosed. Furthermore, Broadbent's objection is raised against bare multifactorial accounts - I do not propose that all there is to a disease is a list of causes, rather, I take diseases to be complexes of causal processes that can be individuated by lists of causes.

With respect to the second of Broadbent's worries (that 'the causes of even the most ordinary event are beyond human counting'): it is indeed pragmatically impossible to know all the causal conditions and their ordering for any one disease, but the practical inability to fully pick out all the identity conditions for a disease does not significantly jeopardise the position. The worry is that diseases become 'unknowable noumena', but this is not an uncommon phenomenon in the sciences. Brian Ellis (2001), as a scientific essentialist, takes the aim of science to be to discover the essential properties of natural kinds, but not knowing all the essential properties of a particular natural kind does not entail that the natural kinds are unknowable, nor does it make Scientific Essentialism wholly unappealing. Just as discovering unknown essential properties of natural kinds refines our scientific knowledge and understanding of the natural world, discovering more possible causes of a disease improves our knowledge, understanding, and ability to manipulate the causal processes it involves. We may have to concede that knowing an individual disease's full cause/identity conditions is beyond the scope of human understanding, but this does not make the position implausible.

\section{Objection $v$}

If one classifies a disease by the complete set of causes that give rise to it, one is vulnerable to there being just one instance of most diseases, as precisely the same event can only be caused in one way, and precisely the same causal conditions are rarely met twice.

Response: Whitbeck argues that a disease is a 'complex of processes' (Whitbeck, 1977, 626), both clinical and pathological. Unless diseases are complexes of pathological and clinical changes, then we risk allowing static conditions like 'physiological or anatomical abnormalities' to count as disease entities (Whitbeck, 1977, 625), and these conditions do not fit well with our conceptual category of diseases.

\footnotetext{
${ }^{8}$ From personal correspondence with Alexander Broadbent, 2013

${ }^{9}$ More on this in section 4.
} 
Whitbeck may not be right to think this. Arguably pathological conditions like aortic stenosis or patent ductus arteriosus (PDA) can cause a specific pathophysiological syndrome which does not have to change in order to be a disease ${ }^{10}$. PDA, a heart defect that occurs when the ductus arteriosus does not close at birth, is prima facie a static condition. Whitbeck might respond, however, that the resultant irregular transmission of blood between the aorta and the pulmonary artery are fundamental aspects of PDA, and of course this is a causal process $^{11}$. Similarly with the former condition, the effects on blood flow of the narrowing of the aortic valve are causal processes. Diseases, at least in general, involve many different kinds of process: temperature-rises, tumour-growth, the deterioration of the immune systems, and so on - but token cases of the same disease need not involve precisely the same changes/causal processes.

Since Aristotle, talk of natural kinds in biology has focused on species/genus classification (LaPorte 2004; Hull 1987; Putnam 1975), but the most persuasive arguments against taxa carving nature at the joints are equally applicable at the 'bodily process' level we associate with diseases. They run roughly as follows:

The properties of every member of a species are the result of tens of thousands of years of evolution. There is little need to go into the details of the evolutionary process here, but this theory (which, if the naturalists are to uphold their commitment to biological theory, they are effectively committed to) is predicated on the fact that the next generation of a species is able to lose or gain any genealogical properties of the previous generation. This has led to the wide acceptance of morphological accounts of species classification, whereby species-membership is determined by 'lists of features, a combination of which is sufficient for kind membership, but no one of which is necessary' (Ruse, 1987, 226).

Evolutionary theory pushes us towards morphological accounts of biological species, but this can also be applied to the functions of their parts. The nature/existence of an individual's heart, and the causal processes associated with it, depends on her genealogical properties. But it is not the case that precisely the same set of causal conditions must be met twice for a process of the same kind to occur twice, after all, lots of people have hearts that are beating - in the case of diseases, assuming diseases to be biological processes, not all symptoms (qua causal processes) associated with a disease need to manifest in order for a patient to suffer from it. As with properties of species, the causal conditions contained within the full cause of a disease is fixed by the list of causal processes associated with it, certain combinations of which are sufficient for kind membership ${ }^{12}$.

\section{A Dispositional Metaphysics of Disease}

Having dealt with these possible objections to CCD, in this section I provide a metaphysic of disease that neatly identifies their most salient features. On the face of it distinct tokens of disease should be classed together in

\footnotetext{
${ }^{10}$ My thanks to an anonymous referee for this observation.

${ }^{11}$ I remain neutral as to whether the abnormal blood flow should be considered an essential part of the disease, or whether one should deem it merely an effect. Either way, even with 'static' conditions, medical practice and research tends to focus on how to prevent or stop the harmful resultant processes.

${ }^{12}$ Note that this claim is equally true for naturalistic and constructivist interpretations of disease.
} 
virtue of the similarity of the clinical and pathological effects of the illnesses, and the conditions that must be satisfied to slow down, cease, or reverse the processes involved - I show here that a dispositionalist ontology nicely captures these ideals.

It is not necessary to delve deep into the dispositional essentialist literature, but crudely, proponents of this position believe that at least some properties have a dispositional essence; dispositional properties dispose their bearers to play certain causal roles; and each disposition has at least one determinate stimulus condition, and at least one determinate manifestation ${ }^{13}$. When an object instantiates dispositional property $\mathrm{P}$ (which disposes it towards manifestation $M$ ), if $\mathrm{P}^{\prime} \mathrm{S}$ stimulus conditions $\mathrm{S}$ are met, then $\mathrm{M}^{14}$. The dispositions of salt and those of water, for example, are such that (in the absence of interfering factors) a saline solution is formed when the two mix. These dispositions are known as 'reciprocal disposition partners' as the solubility of the salt and the water's 'power'/disposition to dissolve salt are equally active properties in the causal process (Martin and Heil 1998). For this, Heil also coins the term 'causing' (Heil, 2013). Dispositionalists thus take causal processes to be the manifesting of dispositional properties, and see all causal interactions as the coming together and mutual manifestation of at least two reciprocal disposition partners ${ }^{15}$. If we view causal processes in this way, a dispositional metaphysics of disease that is useful both theoretically and practically can be developed (Scheuermann et al, 2009; Mizoguchi et al, 2011; Kerry et al, 2012; Eriksen et al 2013).

Individuals are disposed to contract diseases. They possess dispositions triggered by those of intruding diseaseentities, the dispositions of their internal organs ${ }^{16}$, the dispositions of chemical substances, and so on. Epileptics are disposed to have seizures after excessive alcohol consumption; that is to say, alcohol is a stimulus condition for seizures which manifests when consumed by epileptics (in the absence of interfering factors). Some are disposed to go into anaphylactic shock when stung by bees, and smoking is a stimulus condition for the disposition of humans to get lung-cancer. In all these cases there is an initial interaction of the individual's properties and of those of the stimulus (note that sometimes there may be no causal factor originating externally - the stimulus may be some property intrinsic to the human body); these initial causal interactions act as stimulus conditions for the instantiation of further (dispositional) properties, and the causal process continues as the pathological and clinical signs of the disease become more apparent.

Every causal process forming a part of a disease (indeed, any causal process at all!) involves the interaction and manifestation of many different dispositional properties. These manifestations over time include the pathological changes or damage in cell structure at a tissue level, as well as the clinical signs we more readily recognise. Immediately this looks promising for a dispositional account. Consider the type 6 human

\footnotetext{
${ }^{13}$ There are possible counter-examples, such as the decay of a radioactive particle, which seems to have no stimulus, but there is little need to get into this, here.

${ }^{14}$ This might look as though dispositions are reducible to counterfactuals, but there are many reasons to think otherwise (see Mumford and Anjum 2011).

${ }^{15}$ Perhaps with the exception of examples like randomly decaying radioactive particles, but these need not concern us here.

${ }^{16}$ This gives rise to an interesting debate concerning the relationship between the dispositions of the person and the dispositions of her parts, but this is work for another paper.
} 
papillomavirus discussed in section 3. The properties of type $6 \mathrm{HPV}$ (according to the cited sources) can act as stimulus conditions for laryngeal papillomatosis, anal lesions, anogenital warts, oral papillomas, a variety of cancers, and cardiovascular disease. So what determines the pathological changes/changes in cell structure incurred by the patient? One might think it is the circumstances the patient finds herself in - is she in a warm or cold environment? Is she well-hydrated? Does she smoke? Naturally these external factors are causally relevant, but this would be, at best, only a partial explanation: the patient contracts disease D because of her dispositional properties, as well as those of the virus and her environmental conditions. If the patient is disposed not to feel any symptoms upon contracting HPV, she will not feel any symptoms, and if she is disposed to get anal lesions in those circumstances, this will inevitably be the result. Differences in whether two individuals contract a disease in the same circumstances, and what symptoms they suffer if they do, are metaphysically explicable by real differences in their dispositional properties ${ }^{17}$.

Why inevitably? Kerry et al write:

'Because causation involves tendencies towards an outcome, rather than the guarantee of an outcome, then the traditional problem of induction is dissolved. The problem of induction concerned how we could know from past regularities that future cases are guaranteed to be the same. But if we understand causation in dispositional terms, then there should indeed be no rational ground to make that inference. It is indeed always possible that the disposition does not get manifested in some future case'. (Kerry et al, 2012, 1010)

From a strict metaphysical perspective, if the dispositional approach requires the endorsement of the principle outlined above then CCD fails, as the same circumstances would not guarantee the same disease (or any disease at all, for that matter). However one should not be sympathetic to these claims. First, even if causes (merely) tend towards their manifestation, this does not protect the dispositionalist from the problem of induction. How could one know that smoking, tomorrow, will tend towards cancer? Perhaps smoking, tomorrow, will tend towards the reduction of tumour-size! Of course, no rational person would expect smoking to start reducing tumour-growth (no rational person refuses all inductive inferences), but the problem of induction holds as much for Kerry et al as it does for any necessitarian.

Second, this metaphysic precludes CCD only if the effect of a complete set of causal circumstances is indeterministic in a sense stronger than statistical probability. Although there is no 'in principle' reason why Kerry et al might be right in thinking there are 'objective' probabilities, dispositionalism is perfectly compatible with determinism. To say otherwise would be to confuse conceptual analysis with metaphysics. Dispositionalists (see Mumford and Anjum (2011)) often use the properties of ordinary sized objects, such as the fragility of vases and the flammability of matches, to show how a stimulus condition $S$ for disposition $D$ being met does not necessitate its manifestation M. Fragile objects like vases, they say, are disposed to smash

\footnotetext{
${ }^{17}$ From a purely theoretical, metaphysical perspective, to be convincing perhaps one might need to talk about fundamental dispositional properties, and how the arrangement of their instances act as truthmakers for dispositional talk at a non-fundamental level. Reduction of the dispositions of people, organs, and cells to complexes of fundamental property-instances is not necessarily problematic, but more importantly, in the context of philosophy of medicine, these considerations are of little value.
} 
when dropped, but one can interfere post-dropping such that a token vase does not smash by padding the floor with sponges. $S$ being met does not, they claim, necessitate $M$ - indeed they claim there is never necessitation in causation. But if we are to consider rejecting CCD on metaphysical grounds, it better be the case that 'fragility' is a property we should take metaphysically seriously, and this at best a contentious matter. Mumford and Anjum 'do not say, as in Bird (2007), that the only real powers are fundamental. Higher-level properties, such as being fragile, may be messier in a scientific sense, but we need a better reason than that to ignore them' (Mumford and Anjum, 2011, 17), but in doing serious metaphysics we have good reason to do just that. For any token composite object $O$ to be fragile, it must be composed of fundamental, propertied parts, arranged in such a way that the object 'tends towards' smashing. However the inference of propensity without necessity with respect to ordinary sized objects arises from a lack of linguistic precision and an incomplete epistemic picture. The fundamental particles with their fundamental properties will manifest precisely as they should, so Mumford and Anjum's examples provide us with no in principle reason not to think the world is deterministic. In the circumstances, it might well be a matter of metaphysical necessity whether or not the vase will smash, so statistical probability remains sufficient for causation within a dispositional essentialist ontology. As we shall see, in contrast, if we focus on the conceptual rather than on metaphysics and serious ontology, dispositionality being characterised by tendencies (including non-fundamental properties) rather than necessitation is extremely useful, particularly in the context of medical research and practice.

Benefits

(i)

Models of disease call for models of antidotes (that is, substances/procedures that are disposed to stop or reverse the disease processes), and dispositionality is particularly accommodating. For the dispositionalist an antidote introduces new causal conditions such that the previously manifesting dispositions, with their stimulus conditions having already been met, cease to manifest - it 'masks' the manifestation. A diabetic, for example, is disposed to suffer from polyuria, polydipsia, and polyphagia, which manifest when the stimulus condition of ingesting sugar is met, but the injection of insulin changes the conditions so that the patient's blood sugar levels drop and the corresponding symptoms cease to manifest.

Much of modern medicine looks to prevent diseases from manifesting in the first place, so at least three of the main objectives for medical researchers and practitioners can be outlined in dispositional language: first, to look for ways to prevent the stimulus conditions from being met (for example, ensuring people wash their hands to prevent the spread of bacteria in hospitals); second, to look for ways to mask diseases (a neurologist, for example, may prescribe sodium valproate to epileptics in the hope that it will mask their disposition to have seizures.); and third, to provide antidotes to the disease to halt its manifestation, that is, to relieve the symptoms (paracetamol to relieve pain), and prevent the continued progression of the unwanted causal processes (antibiotics to stop the processes associated with harmful bacterial infections). 
(ii)

DMD affords a more transparent account of explanatory 'saliency' in the philosophy of medicine. Epidemiologists, lawyers, and insurance companies (not to mention patients!) are most interested in what could have been done to prevent the disease the patient suffered from, the likely consequences of its being contracted, and what might be done or could have been done to cure it. With the dispositional analysis, one can take the salient causes not necessarily to be those that make the greatest causal contribution ${ }^{18}$ to the effect (the disease, or perhaps the consequences of the disease), but those causal factors we can either prevent (the stimulus conditions) or ' mask' $^{19}$.

One might object that this notion of saliency identifies tonsils as a salient cause of tonsillitis, since we have these removed to prevent the disease (surely tonsils cannot be a cause of tonsillitis!), but on inspection this is not troublesome - one of the causes of tonsillitis is our disposition to get tonsillitis, a property instantiated by all and only those of us with tonsils. To prevent tonsillitis we can remove our disposition to contract the disease by removing our tonsils. It is the individual who suffers from tonsillitis, not the tonsils, so to suppose that properties of the tonsils dispose us towards, and hence act as a cause of tonsillitis, is not unreasonable.

Of course there are far more dispositions involved in the development of a disease than those we consider most salient. Without their reciprocal disposition partners, the dispositions we consider most salient, say, the dispositions of the flu virus, cannot manifest, and many of these reciprocal disposition partners are property instances necessary for the body's healthy functioning. The flu virus cannot cause suffering without causal interaction with previously 'healthy' cells. Arguably, so far as Nature is concerned, the dispositions of the healthy cells are equally causally active in causing harm as those of the virus. This should not be considered problematic for the dispositional conception, however, as interfering with perfectly healthy patients can, as is the case with vaccinations, help prevent disease.

(iii)

The cases considered in this paper have consisted exclusively of physical ailments, but many mental disorders are considered diseases. On the face of it these do not fit in so well with the metaphysic I am proposing. Take Rich, who applied to but failed to get a place at Cambridge University, and as a result became very depressed. Sam, Rich's twin, was turned away from Cambridge in precisely the same way as Rich. Sam decided she would

\footnotetext{
${ }^{18}$ Take the cause that makes 'greatest causal contribution' to be the cause that, individually, raises the probability of contracting the disease by the greatest amount.

19 'Masking' the properties initiating disease-processes, or in other words, removing the causal base of a disease, is something we do on a very regular basis. Note that it also seems reasonable to suppose the absence of, say, a vaccination; that is, the non-introduction of a mask, can be a conceptually salient causal factor in contracting a disease. Again, this fits well with the Millian causal classification model provided above (as we saw with the 'scurvy' case, the absence of some factor is often considered the most salient causal factor).
} 
make the most of the situation. She re-sat her A-Levels in time to secure her place at Oxford, and was all the while in fairly good spirits. So how does one explain the very different reactions?

The obvious response (in the context of this paper!) is that Rich is disposed towards depression and Sam is not, so when the triggering conditions were met only Rich became depressed. But this is not a particularly informative, scientific explanation. Although unsuccessful thus far, researchers are currently looking for genealogical links with depression (Hek et al, 2012). Significant results would provide a useful test for discovering whether a patient is genetically disposed to suffer from depression, but research concerning the triggering conditions cannot be tested in the same way. Take the cause of Rich's depression: Rich's state of mind was badly affected by his experiencing a succession of stressful situations, but on the face of it nothing physical interacted with Rich's dispositional properties. The dispositions of a virus mutually manifest with the dispositions of biological cells, but the relationship between Rich's state of mind and the stressful situations is prima facie unlike this. The dispositions of Rich's brain do not mutually manifest with the dispositions of the stressful situation, as stressful situations are metaphysically not the kind of thing that can do this. In short, stressful situations may well be disposed to induce states of depression, but Rich's disposition to get depression cannot causally affect the stress-inducing properties of the situation (these do not change).

This does not, however, require us to have a different metaphysical attitude toward mental disorders as we do to 'physical' diseases - mental states, malfunctioning or otherwise, are all intimately linked with brain states ${ }^{20}$. The changes in brain state, here; that is, the physical changes associated with depression, can be treated just as any other physical ailments - we can and do use chemicals to mask certain disposition manifestations. Furthermore, we have good reason to conceptualise mental disorders in precisely this way. Although it is not within the scope of this paper to investigate this issue in detail, looking at mental disorders in terms of dispositions is very intuitive. One might plausibly argue that to help a mentally ill patient with their mental disorder, just is to manipulate their dispositions such that they no longer respond to stimuli in ways detrimental to the health of themselves or others.

\section{Disease Processes versus Non-Disease Processes}

I have not attempted to distinguish disease-processes from non-disease-processes, though clearly this is a conceptual question of interest and importance. Here I demonstrate that CCD is compatible with both the naturalist and constructivist conceptual analyses of disease.

The naturalists believe diseases can be accurately described only by using the language of science, and it is only through the use and development of scientific/biological theory that research into diseases can be conducted. Boorse (a naturalist) claims that the functioning of a human body comprises myriad complex natural biological processes, and diseases are just those natural bodily processes that malfunction (Boorse 1997). For Boorse, disease-talk concerns the natural function of the relevant bodily process, the way in which it is malfunctioning, and the explanation for its malfunctioning.

${ }^{20}$ I shall leave it to those philosophers of mind to argue over how! 
For the constructivist, conversely, biological processes have no ontologically significant 'natural' role ${ }^{21}$, so to say that a natural biological process can malfunction is (on the face of it) nonsensical (unless we take 'natural' to be a predicate we assign to those processes we associate with good health, but of course this would be a constructivist conception of the term). For the constructivist 'disease' is a normative notion whereby we make judgements about certain kinds of harm caused by biological processes. Diseases are not objectively 'unnatural', or malfunctioning natural processes, they are harmful biological processes we have deemed incompatible with our conception of 'normally' functioning, healthy human beings, but there need not be any reference to 'malfunctioning natural processes'. Consider two skilled physicians from different socioeconomic backgrounds examining the same patient. It is far from inconceivable that they make different diagnoses, purely as a result of their different backgrounds. Even if these diagnoses are incompatible (suppose one said their patient had a disease, and the other denied this claim) for the constructivist they could both be correct, given the socioeconomic contexts in which those judgements were made. For most, then, modern medicine seems distinctly anti-constructivist (whether one has heart disease is thought to be a matter entirely independent of socioeconomic conditions), but that is not to say it should be!

If one is a naturalist about disease, there is a fixed list of diseases that can be correctly individuated by CCD. If one is a constructivist, at any one time there is an ideal list of diseases (fixed by our socioeconomic circumstances), whether we know the content of this list or not - again, these can be individuated by CCD. In either case, CCD is a good means of individuating diseases; it provides an explanation for the symptoms patients with the disease suffer from, and it grounds the process of selecting treatments.

\section{Causal Classification and Dispositional Models of Disease Reconciled}

In keeping with CCD it is not the case that just one cause (qua single dispositional property-instance) is responsible for a disease. The central claim of CCD is that a disease can be individuated by a disjunction of conjunctions of causes. DMD states that from an ontological point of view, diseases are constituted by the simultaneous manifestation of reciprocal disposition partners (these always concern the dispositions of the patient, and often those of an external agent - perhaps most obvious in the case of infectious diseases). According to the CCD+DMD model, then, diseases have a 'full cause' to be cashed out in terms of the distribution of many dispositional property instances, so we can individuate a disease in terms of the possible circumstances/distributions of property instances that might cause the disease in question. From a metaphysical perspective, the dispositions of the patient are causally active in precisely the same way as those of external factors, so these have a rightful place within disease classification. CCD can be expressed equally well in dispositionalist terms as it can in 'standard' event-talk.

\section{Conclusions}

Regardless of whether one is happy to end one's analysis of causation with talk of counterfactuals and conditionals, or whether one wishes to explore the numerous necessitarian metaphysics of causation,

\footnotetext{
${ }^{21}$ This is perhaps an over-generalisation, but most constructivists would endorse such a claim.
} 
conditionals can be used to individuate individual diseases, since of necessity, no two diseases can have the same complete set of possible circumstances sufficient for their contraction; CCD is thus an ideal model for disease-individuation.

Bodily processes involve the coming together of reciprocal disposition partners and the ongoing manifestation of properties. Sometimes we deem these processes to be conducive to healthy bodily functioning, sometimes these manifestations cause (or indeed 'are') pain, suffering, a shortening of life-expectancy, and other such undesirable consequences. All diseases are complexes of these dispositional processes. Of course, not all harmful bodily processes are cases of disease - were I to be shot there might be bodily processes that ultimately lead to my death, but these are not disease-processes. However this is work for another paper, as untangling the details of which complexes of processes count as diseases and which do not is an issue independent both of what diseases are from a metaphysical (as opposed to a conceptual analysis) perspective, and how one can individuate the diseases we have $\mathrm{e}^{22}$.

Dispositional talk is extremely useful in a medical context, as notions of masking dispositions should be fundamental to medical practice and research. Furthermore, viewing disease both in terms of the dispositions of the patient and those of any other etiological agent (for example, bacteria or viruses in the case of infectious diseases) is important, as each side of the story is as important as the other. One should remember from the discussion of the underlying metaphysics, though, that the indeterminacy in medicine might strictly only be apparent. The more refined our knowledge of the 'actual' dispositions of the patient and those of the 'actual' circumstances in which the patient becomes diseased, the more deterministic the contraction and curing of disease is likely to look.

Acknowledgements: I would like to thank Alexander Broadbent, Kengo Miyazono, Michael Talibard, and two anonymous referees for their comments on earlier versions of this paper.

\section{Bibliography}

Bird, A

(2007) Nature's Metaphysics: Laws and Properties Oxford Clarendon Press

Broadbent, A

(2009) 'Causation and Models of Disease in Epidemiology' Studies in History and

Philosophy of Biological and Biomedical Sciences 40 302-311

\footnotetext{
${ }^{22}$ My intuition is that the distinction is to be made in terms of the malfunction of bodily processes - this is generally entailed by the naturalistic accounts, but I take it that those opposed to this model need to provide a constructivist account of bodily malfunction.
} 
(2012) "Causes of causes." Philosophical studies 158.3: 457-476.

(2013) The Philosophy of Epidemiology Palgrave, Macmillan

Boorse, $\mathrm{C}$

(1975). “On The Distinction Between Disease and Illness," Philosophy and Public

Affairs, 5: 49-68.

(1976) "What A Theory of Mental Health Should Be," Journal for the Theory

of Social Behavior, 6: 61-84.

(1977) "Health as a Theoretical Concept," Philosophy of Science, 44: 542-

573.

(1997) “A rebuttal on health," in J. M. Humber and R. F. Almeder

(Eds.), What is disease? Totowa, NJ: Humana Press, 3-143.

Cohen, J. and C. Callender. (2009). A better best system account of lawhood, Philosophical Studies 145:1-34.

Collins, J., Hall, E. E. J., \& Paul, L. A. (Eds.). (2004). Causation and counterfactuals. The MIT Press.

Ellis, B (2001) Scientific essentialism. Cambridge University Press

- (2009) TheMetaphysics of Scientific Realism Durham: Acumen Publishing

Ellis, B \& Lierse, C $\quad$ (1994) 'Dispositional Essentialism' Australasian Journal of Philosophy 72 (1):27 - 45

Eriksen, T et al (2013) At the borders of medical reasoning: aetiological and ontological challenges of medically unexplained symptoms Philos Ethics Humanit Med. 2013; 8: 11.

Hek, K et al. (2012) A genome-wide association study of depressive symptoms. Biological Psychiatry. doi: 10.1016/j.biopsych.2012.09.033

Heil, J. $\quad$ (2013) The Universe as We Find It Oxford University Press

Hull, D. L. (1987) ‘Genealogical Actors in Ecological Roles’, Biology and Philosophy 2: 168-184.

Johnston, M (1992) 'How to Speak of the Colors', Philosophical Studies 68: 221-263.

Kerry R et al (2012) Causation and evidence-based practice: an ontological review. Oct; 18(5):1006-12. doi: 10.1111/j.1365-2753.2012.01908.x.

Kuo, HK; Fujise, K (2011-11-01). 'Human papillomavirus and cardiovascular disease among u.s. Women in the national health and nutrition examination survey, 2003 to 2006.'. Journal of the American College of Cardiology 58 (19): 2001-6 
LaPorte, J.

(2004) Natural Kinds and Conceptual Change. Cambridge: Cambridge University

Press

Riichiro Mizoguchi et al (2011) River Flow Model of Diseases. ICBO International Conference on Biomedical Ontology

Lewis, D.

(1987) Philosophical Papers // Oxford University Press (first published (1973)

“Causation", Journal of Philosophy, 70: 556-67.)

- $\quad$ (1973) Counterfactuals. Oxford: Blackwell Publishers and Cambridge: Harvard University Press, 1973

- $\quad$ (2000) Causation as influence. The Journal of Philosophy 97.4: 182-197.

- $\quad$ (1994). Humean supervenience debugged. Mind 103:473-490.

Mackie, J.L.

(1974) The Cement of the Universe Oxford Clarendon Press

Martin, CB; Heil, J

(1998) "Rules and Powers" Noûs Volume 32, Issue S12, pages 283-312, October 1998

Millikan, R.G. (1984). Language, Thought, and Other Biological Categories. MIT Press, Cambridge.

Muñoza et al

(2006) "Chapter 1: HPV in the etiology of human cancer". Vaccine 24S3 S3/1-S3/10

Mumford, S

(2004) Laws in Nature Routledge

Mumford, S and Anjum, R (2011) Getting Causes from Powers

Oxford University Press

Murphy, D. (2006) Psychiatry in the Scientific Image, Cambridge, MA: MIT Press.

- "Concepts of Disease and Health", The Stanford Encyclopedia of Philosophy (Summer 2009 Edition), Edward N. Zalta (ed.), URL = <http://plato.stanford.edu/archives/sum2009/entries/health-disease/>.

Neander, K. (1991). The teleological notion of 'Function'. Australasian Journal of Philosophy 69: 454-468.

Putnam, $\mathrm{H}$. (1975) Mind, Language and Reality: Philosophical Papers Vol. 2 Cambridge University Press.

Reichenbach, $\mathrm{H}$. (1949) The Theory of Probability. University of California Press 
Ruse, $\mathrm{M}$

(1987) "Biological Species: Natural Kinds, Individuals, or What? The British Journal for the Philosophy of Science Vol.38 No.2 (June, 197) pp225-242

Salmon,W. C.

(1967) The Foundations of Scientific Inference University of Pittsburgh Press

Schaffer, J.

(2003) “Contrastive Causation” The Philosophical Review Vol. 114, No.3 (July 2005),

pp327-358

Scheuermann et al (2009) Toward an Ontological Treatment of Disease and Diagnosis Summit on Translat Bioinforma 2009: 116-120. Published online March 1, 2009.

Schrenk, M.

(2010) The Powerlessness of Necessity Nous, 44, (4), pp 725-739

Smart, BTH

(2013) Is the Humean Defeated by Induction Philosophical Studies 162 (2), 319-332

Whitbeck, C.

(1977) Causation in Medicine: The Disease Entity Model, Philosophy of Science, 44,

619-637.

Whitbeck, C.

(1981) A Theory of Health, in A. L. Caplan and H. T. Engelhardt, Jr. (eds.), Concepts of Health and Disease: Interdisciplinary Perspectives, Reading, MA: Addison-Wesley: 611-626

World Health Organization (WHO)

(1948) WHO definition of Health, in Preamble to the Constitution of the World Health Organization as adopted by the International Health Conference, New York, 19-22 June, 1946; signed on 22 July 1946 by the representatives of 61 States (Official Records of the World Health Organization, no. 2, p. 100)

World Health Organization (WHO)

(1980) Bulletin of the World Health Organisation, 58 (4): 585-591 